\title{
Focused ion beam modification of surfaces for directed self-assembly of InAs/GaAs(001) quantum dots
}

\author{
Hugh McKay, Paul Rudzinski, Aaron Dehne and \\ Joanna Mirecki Millunchick
}

Department of Materials Science and Engineering, University of Michigan, Ann Arbor, MI 48109, USA

Received 12 July 2007, in final form 11 September 2007

Published 10 October 2007

Online at stacks.iop.org/Nano/18/455303

\begin{abstract}
Controlled nucleation of InAs quantum dots has been achieved by $\mathrm{Ga}^{+}$ focused ion beam modification of $\mathrm{GaAs}(100)$ surfaces. Quantum dots may be induced in irradiated regions despite the fact that the deposited thickness is less than the critical thickness for their formation under typical growth conditions when the ion dose is greater than $10^{13}$ ions $\mathrm{cm}^{-2}$. We also find that the dot density increases with increasing ion dose, and reaches saturation for $D>10^{14}$ ions $\mathrm{cm}^{-2}$. Parameters such as dot height and diameter are unaffected by the dose level. Thus, we show that the increase in dot density is a result of diffusion of adatoms from outside the patterned region. The mechanism for enhanced quantum dot formation is due to the formation of monolayer deep holes created in the substrate by the ion beam, which may be used to form regular arrays of quantum dots.
\end{abstract}

\section{Introduction}

InAs quantum dots are of interest for optoelectronic devices in the important $1.3-1.55 \mu \mathrm{m}$ telecom optical fiber window $[1,2]$ and show strong potential for discrete photon emitters [3]. There are numerous schemes to control the nucleation locations of the dots [4], ranging from standard lithographic techniques $[5,6]$ to atomic force lithography $[7,8]$ and patterning using a scanning tunneling microscope [9]. Recently, focused ion beams (FIBs) have been used to direct the nucleation of $\mathrm{Ge}$ dots in the $\mathrm{Ge} / \mathrm{Si}(001)$ system [10-12]. In that work, FIB directed nanostructures show promise for computing applications in the form of quantum cellular automata. However, since it is based around a material with an indirect band gap, photonic applications are unlikely.

FIB patterning [9] and subsequent self-assembly of quantum dots $[13,14]$ has also been demonstrated for the IIIV compound semiconductors. Sun et al obtained continuous lines of InP on FIB patterned GaAs [13], while Morshita et al obtained discrete InAs dots that have nucleated along the side walls of FIB patterned holes in GaAs [14]. As such, FIB patterning has emerged as a viable tool for the fabrication of photonic and optoelectronic devices $[15,16]$. The mechanisms for the preferential assembly of these features on ion-induced patterns is still in question. In this paper, we examine the effect of a rastered ion beam on the assembly of InAs quantum dots. We show that the mechanism that governs the FIB directed self-assembly of quantum dots on these broadly dosed regions is the formation of surface holes by the ion beam that act as a sink for In adatoms.

\section{Experimental details}

Samples were grown using an EPI 930 molecular beam epitaxy system on $\mathrm{GaAs}(001)$ substrates. Typical values for growth rates were $R_{\mathrm{Ga}}=0.75$ monolayers $/$ second $\left(\mathrm{ML} \mathrm{s}^{-1}\right), R_{\mathrm{In}}=$ $0.25 \mathrm{ML} \mathrm{s}^{-1}, R_{\text {As4(low) }} \sim 1.0 \mathrm{ML} \mathrm{s}^{-1}$ and $R_{\text {As4(high) }} \sim$ 2.2 $\mathrm{ML} \mathrm{s}^{-1}$. Two different $\mathrm{As}_{4}$ fluxes were employed to give similar III/V flux ratios while growing GaAs or InAs. After oxide desorption, an $h=400 \mathrm{~nm}$ GaAs buffer layer was grown at a substrate temperature of $T=580^{\circ} \mathrm{C}$ under high $\mathrm{As}_{4}$ flux. Following buffer layer growth, the sample was annealed at $T=$ $580^{\circ} \mathrm{C}$ under high $\mathrm{As}_{4}$ flux and then allowed to cool to room temperature under low arsenic flux. When cool, the sample was transferred under vacuum to a chamber attached to the growth system containing a FEI Magnum FIB system. The FIB was employed to treat $5 \mu \mathrm{m} \times 5 \mu \mathrm{m}$ squares with increasing ion doses $D$ in the range $1 \times 10^{12}<D<8 \times 10^{16}$ ions cm $\mathrm{cm}^{-2}$, 
(a)

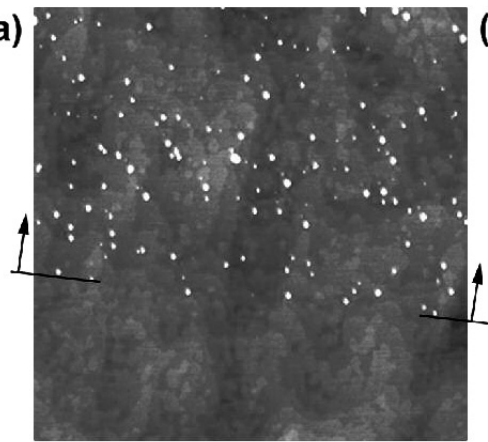

(c)

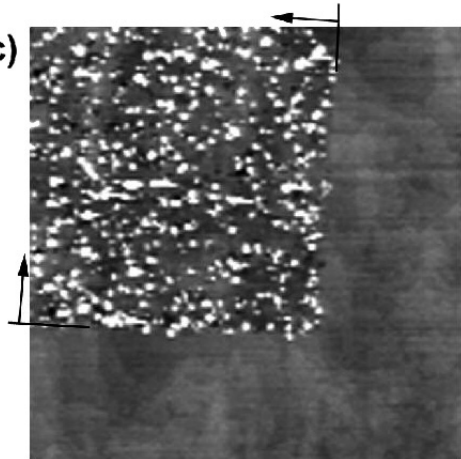

(b)

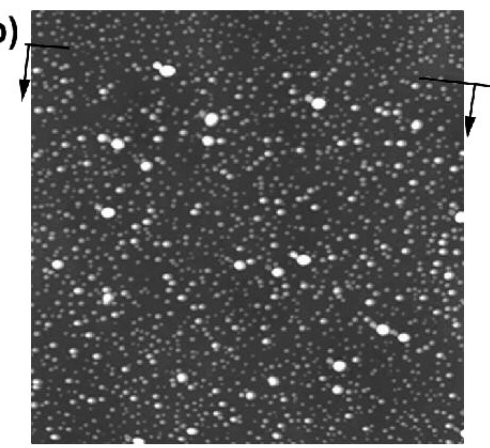

(d)

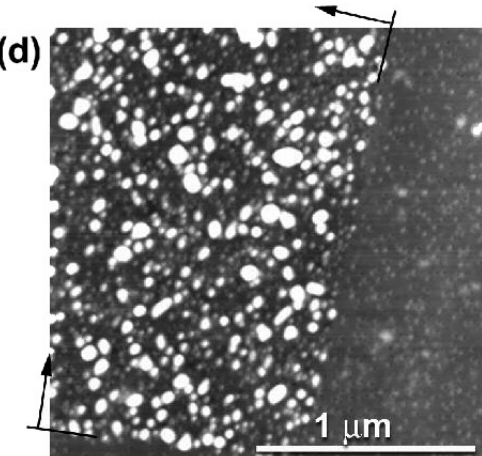

Figure 1. AFM micrographs of $h=2.0\left(\right.$ (a) and (c)) and 2.4 ML ((b) and (d)) thick InAs on GaAs substrate irradiated with $6.1 \times 10^{13}$ ((a) and (b)) and $5.6 \times 10^{14}$ ions $\mathrm{cm}^{-2}((\mathrm{c})$ and $(\mathrm{d}))$. The hash marks and arrows indicate the regions that were treated with the ion beam. The height scale is (a), (b) $10 \mathrm{~nm}$, (c), (d) $22 \mathrm{~nm}$.

giving predicted sputter depths of 0.004-14 ML [17]. In each $5 \mu \mathrm{m} \times 5 \mu \mathrm{m}$ area, the pixel spacing was $\sim 30 \mathrm{~nm}$, which is smaller than the ion beam size, with the pixels arranged in a square 169 pixels on a side. Each square was dosed in a single pass with a $30 \mathrm{keV}, 7.5 \mathrm{pA}$ beam moving down the square in a serpentine fashion. After treatment, samples were returned to the growth chamber under vacuum and the sample temperature was raised to $T=530^{\circ} \mathrm{C}$. Because the sample never left the vacuum system, issues often observed with regard to preferential oxidation due to ion irradiation $[18,19]$ are circumvented. Thin layers of InAs $(1.7 \leqslant h \leqslant 2.4 \mathrm{ML})$ were deposited at $T=530^{\circ} \mathrm{C}$ under a low arsenic flux while the surface had a $\mathrm{c}(4 \times 4)$ reconstruction. Following growth, samples were quenched to room temperature under low $\mathrm{As}_{4}$ flux. Samples were characterized ex situ using a Digital Instruments Nanoscope III atomic force microscope (AFM) in tapping mode.

\section{Results and discussion}

The growth of a thin lattice mismatched layer on a substrate irradiated by FIB results in an apparent reduction of the critical thickness for quantum dot formation. Figure 1 shows a series of AFM micrographs of InAs films grown at $T=530^{\circ} \mathrm{C}$ and $R_{\mathrm{In}}=0.25 \mathrm{ML} \mathrm{s}^{-1}$ and $R_{\mathrm{As}}=1.0 \mathrm{ML} \mathrm{s}^{-1}$ on $\mathrm{GaAs}(001)$ that were patterned by the FIB at various ion beam doses. In this AFM image, no quantum dots are apparent outside of the dosed region but do appear within the patterned areas for $h=2.0$ ML. Furthermore, the dot density is higher for higher ion doses. For a greater thickness, $h=2.4 \mathrm{ML}$, quantum dots develop across the entire surface, but have a higher density and size distribution within the irradiated regions. Thus, we demonstrate that the critical thickness for quantum dot formation is reduced by FIB patterning. For these growth and patterning conditions, the average diameter of self-assembled quantum dots outside the dosed regions is $29 \mathrm{~nm}$, with an average height of $5 \mathrm{~nm}$. Figure 2 shows a plot of the average diameter (figure 2(a)) and average height (figure 2(b)) for film thickness $1.7 \mathrm{ML} \leqslant h \leqslant 2.4 \mathrm{ML}$ and various ion beam doses. These plots demonstrate that, although the average diameter and height are somewhat less than those of quantum dots assembled in the absence of ion irradiation, the standard deviations (denoted by the error bars) are larger for these growth and patterning conditions.

Examination of the behavior of quantum dot formation as a function of thickness deposited and ion beam dose shows that the quantum dot density increases with increasing dose, a trend that is robust over three orders of magnitude in the ion beam dose. Figure 3 shows a plot of the quantum dot density $N$ as a function of ion beam dose $D$ and film thickness $h$. The density of the dots is altered for doses $D>10^{13}$ ions $\mathrm{cm}^{-2}$. Below this dose, there is no discernable change in the quantum dot density within the treated region compared to the untreated areas, in agreement with results for similar experiments on FIB-directed assembly of Ge quantum dots on Si [20]. For $h=1.7$ and 2.0 ML, there were very few quantum dots observed in the undosed regions. Given the size of the AFM micrographs gathered, the maximum dot density is estimated to be $N<$ $2 \times 10^{7} \mathrm{~cm}^{-2}$ for these samples. For $D>10^{13}$ ions cm ${ }^{-2}$, $N$ increases with increasing $D$ up to some maximum density for all the thicknesses examined in this study. The thickest films reach the maximum density more quickly than the thinner 

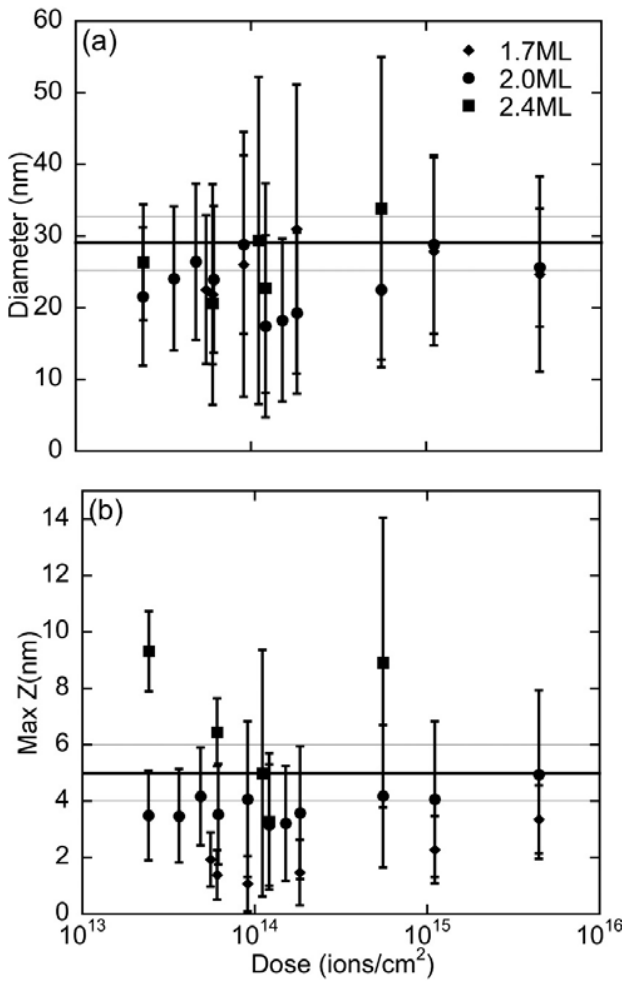

Figure 2. Plot of the (a) average diameter and (b) average height for $1.7 \mathrm{ML} \leqslant h \leqslant 2.4 \mathrm{ML}$ and $10^{13}<D<10^{16}$ ions $\mathrm{cm}^{-2}$. The heavy and thin lines denote the average and standard deviation values for quantum dots assembled in the absence of ion irradiation.

films, and the saturation density increases with increasing deposited thickness. For $h=2.4 \mathrm{ML}$, the maximum dot density $N$ is $N=4.7 \times 10^{10} \mathrm{~cm}^{-2}, N=2.9 \times 10^{10} \mathrm{~cm}^{-2}$ for $h=2.0 \mathrm{ML}$, and $N=1.4 \times 10^{10} \mathrm{~cm}^{-2}$ for $h=1.7 \mathrm{ML}$. The maximum dot density increases with increasing deposited thickness simply due to the fact that the amount of material available for the formation of quantum dots is greater for larger thickness. Despite this increase in the dot density, the quantum dot diameters and heights do not vary with the ion dose or thickness deposited, as can be seen in figure 2 .

The fact that the size and height of the quantum dots does not change with increasing ion beam dose despite an increasing dot density suggests two possible mechanisms. The first is that more $\mathrm{Ga}$ adatoms from the substrate are available to participate in the nucleation of the dots, perhaps due to increased step edge density induced by the ion beam. In this instance, FIB-induced quantum dots would have a lower In content than self-assembled dots. More likely, however, the increase in quantum dot density may be caused by In adatoms diffusing from outside the treated region. Evidence of enhanced diffusion from outside the dosed region is shown in figure 4, which shows an AFM image of an $h=1.7 \mathrm{ML}$ InAs film in the vicinity of the $D=1.1 \times 10^{15}$ ions cm $^{-2}$ irradiated region. For this somewhat higher dose, significant roughening within the treated region is apparent, in addition to quantum dot formation. Because the thickness of the film is less than the critical thickness for quantum dot formation, there are no quantum dots outside the dosed region visible in the micrograph. The step structure is discernable outside

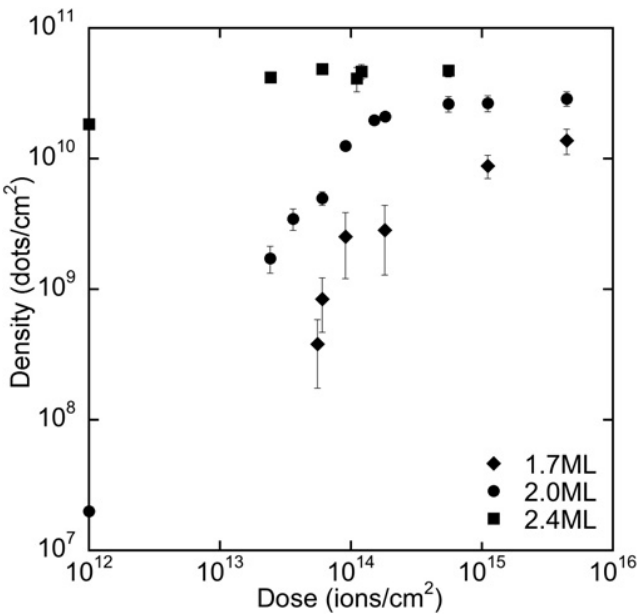

Figure 3. Plot of the quantum dot density $N$ as a function of ion beam dose $D$ and film thickness $h$.

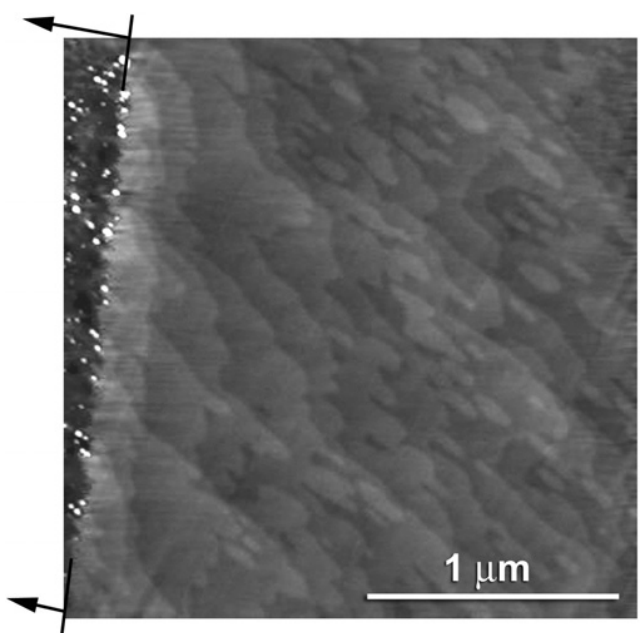

Figure 4. AFM image of a 1.7 ML InAs film in the vicinity of a region irradiated at $D=1.1 \times 10^{15}$ ions $\mathrm{cm}^{-2}$. The hash marks and arrows indicate the region that was treated with the ion beam. The height scale is $5 \mathrm{~nm}$.

the irradiated region, while two dimensional (2D) islands and terraces are clearly observed far from the treated region. However, the terraces are denuded of 2D islands within $1 \mu \mathrm{m}$ of the dosed region in this image, suggesting that the diffusion length of adatoms on this surface is on the order of $1 \mu \mathrm{m}$. Averaged over all irradiated regions for deposited thicknesses of InAs less than the critical thickness for quantum dot formation, the denuded zone is $\approx 0.75 \mu \mathrm{m}$ and does not vary with ion beam dose. In this case, a variation in dot density across the $5 \mu \mathrm{m}$ treated regions would be expected. That is, the density of quantum dots should be larger near the edges of the treated regions than in the center if only In adatom diffusion were responsible for the increase. Such variation is not apparent in the $5 \mu \mathrm{m}$ regions in these experiments. Thus, it is possible that other factors, such as an enhanced Ga adatom density, may be contributing to the increase in the density of quantum dots in the treated regions. It is interesting to note, however, that for thicknesses of InAs greater than the critical 

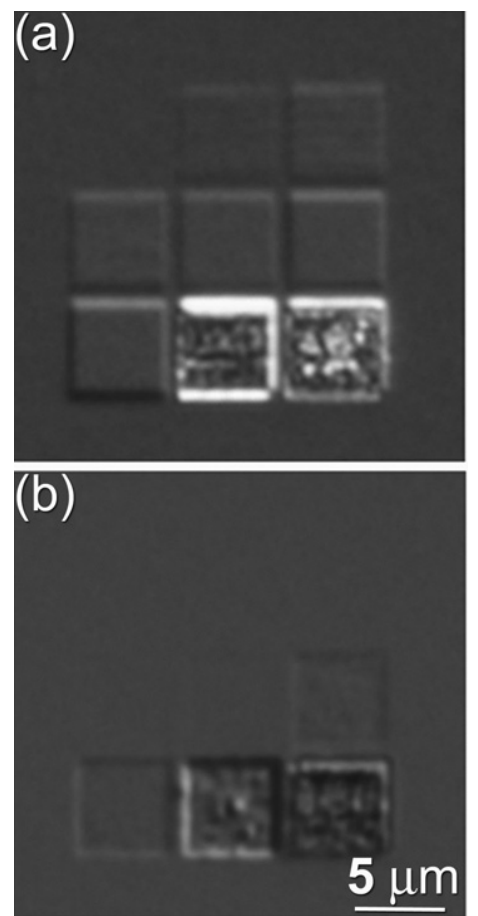

Figure 5. Nomarski micrographs of GaAs buffer layers that have been (a) irradiated with (top row from left to right) $0.8,3.8$, $7.7 \times 10^{13}$ ions cm ${ }^{-2}$, (center row) $3.8,7.6,38.3 \times 10^{14}$ ions $\mathrm{cm}^{-2}$, (bottom row) $7.6,38.3,76.5 \times 10^{15}$ ions $\mathrm{cm}^{-2}$, and (b) irradiated and annealed to $T=580^{\circ} \mathrm{C}$.

thickness there is no region denuded of quantum dots (see, for example, the $h=2.4 \mathrm{ML}$ films in figure 1). This suggests that the nucleation rate of dots is faster than the rate of adatom diffusion for these films. This observation is in direct contrast to what is observed in the $\mathrm{GeSi} / \mathrm{Si}$ system, where there is a denuded zone that increases in size as a function of ion dose regardless of the thickness of the deposited layer [20].

Several mechanisms by which FIB patterning induces quantum dot nucleation have been proposed for the $\mathrm{SiGe}$ system, some of which may or may not be valid for the III-Vs. One is that the $\mathrm{Ga}^{+}$ions alter nucleation through a surfactant-like mechanism [20]. It is doubtful that for very low doses the presence of free Ga on the surface alters the nucleation in these experiments, as any free Ga would be converted to GaAs upon annealing the surface under an As overpressure prior to the deposition of InAs. Nor have we seen any evidence of contamination of the surface by Ga droplets, as have been observed at higher ion doses and energies $[9,18]$. On the other hand, ion beam induced surface or sub-surface damage in the form of vacancies and interstitials may influence the heterogeneous nucleation of quantum dots. Ion-induced oxidation of the surface [19], however, is not a likely mechanism, as the samples remain under high vacuum conditions throughout the patterning and growth process.

If sub-surface damage is a major instigator in FIB directed assembly of quantum dots, then nucleation will proceed even after the damaged areas have been buried. Additionally, high temperature anneals following ion patterning mitigate sub-surface damage and reduce the probability of directed quantum dot nucleation [21]. Figure 5 shows a set of

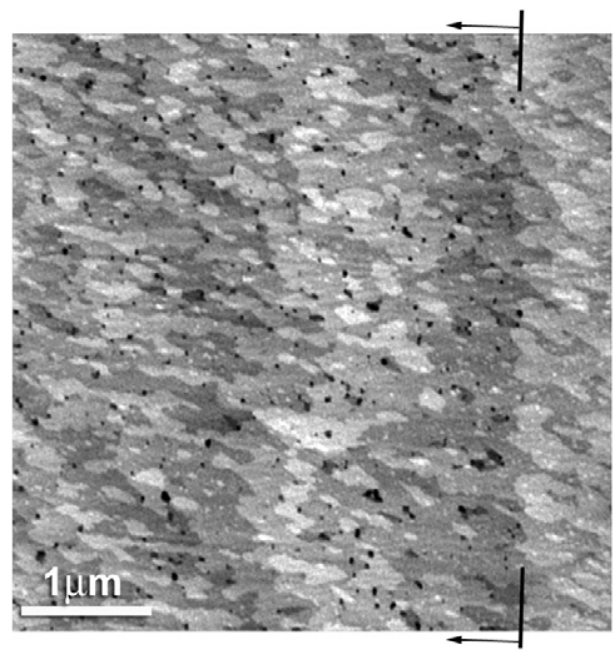

Figure 6. AFM of GaAs that has been irradiated with $1.11 \times 10^{14}$ ions $\mathrm{cm}^{-2}$ and annealed to the growth temperature of InAs. The hash marks and arrows indicate the region that was treated with the ion beam. The height scale is $4 \mathrm{~nm}$.

Nomarski micrographs of a $\mathrm{GaAs}(001)$ substrate that have been patterned with ion doses ranging from $7.7 \times 10^{12}<$ $D<7.7 \times 10^{16}$ ions $\mathrm{cm}^{-2}$ (figure 5(a)), and a similarly patterned region that has been annealed at $T=580^{\circ} \mathrm{C}$ (figure 5(b)). The contrast observed for the regions irradiated with $D<5 \times 10^{14}$ ions $\mathrm{cm}^{-2}$ pictured in figure 5(a) is not related to variations in height, as AFM images of these regions (not shown) do not indicate appreciable changes in the height profiles. Therefore, we propose that the contrast in these regions arises due to changes in the index of refraction induced by the ion beam alone. Annealing the surface largely removes this contrast for the regions irradiated with $D<$ $5 \times 10^{14}$ ions $\mathrm{cm}^{-2}$ (figure 5(b)). The contrast in figure 5 (a) for the regions dosed with $D>5 \times 10^{14}$ ions $\mathrm{cm}^{-2}$ results from both a difference in the height due to sputtering within this region, and to ion induced changes in the index of refraction. Upon annealing, the ion induced damage is removed, and the remaining contrast is predominantly a result of variations in height due to sputtering.

Surface damage in the form of surface vacancies or roughness created by the ion beam may be another mechanism by which the ion beam induces quantum dot nucleation, as quantum dots have been observed to nucleate preferentially on regions of high step density $[13,22,23]$. In the case of the experiments presented here, regions of increased step density are created when the ion beam roughens the surface and forms surface holes. Figure 6 shows an AFM image of a $\mathrm{GaAs}(001)$ buffer layer that has been irradiated with a dose of $D=1.11 \times 10^{14}$ ions $\mathrm{cm}^{-2}$, and returned to the MBE chamber without breaking ultra-high vacuum to be annealed to $T=530^{\circ} \mathrm{C}$, the growth temperature for InAs growth. No InAs was grown, however, as the purpose was to examine the surface morphology prior to quantum dot nucleation. For the $D<10^{13}$ ions $\mathrm{cm}^{-2}$, there is very little change to the surface, and the irradiated region is difficult to discern from the untreated areas according to AFM (not shown). For higher doses, the step structure is altered as can be seen in figure 6 , which is an AFM of a GaAs substrate that has been irradiated 

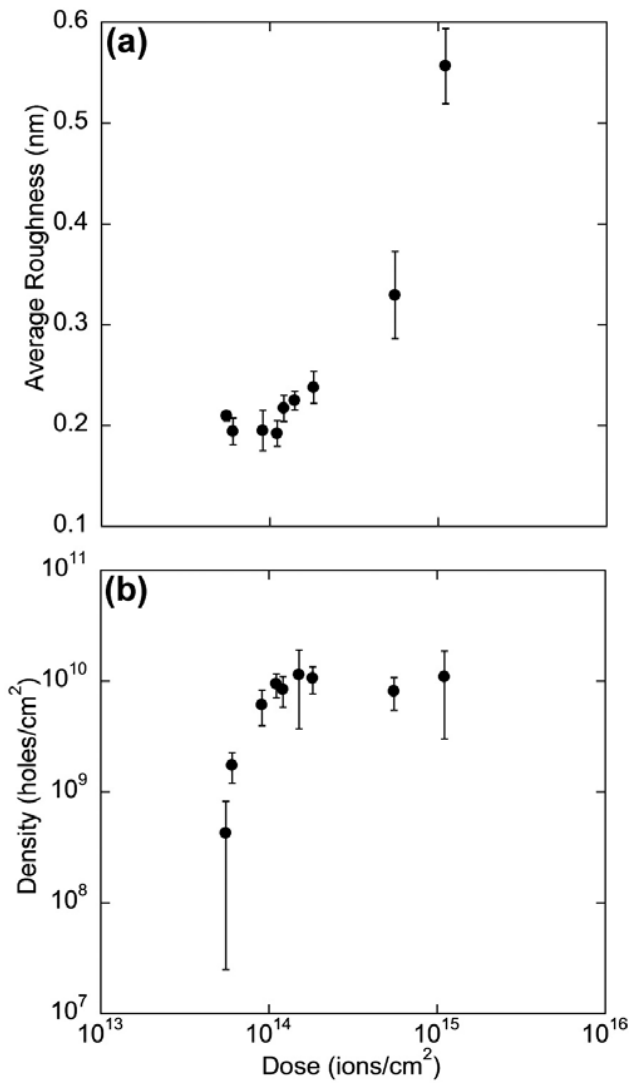

Figure 7. Plot of the (a) average roughness and (b) hole density in $\mathrm{GaAs}(001)$ that has been patterned at various doses and annealed to the growth temperature of InAs.

with $D=1.11 \times 10^{14}$ ion $\mathrm{cm}^{-2}$ and annealed. The irradiated region is bounded by monolayer high steps, and the interior is characterized by increased roughness in the form of $2 \mathrm{D}$ islands and the development of holes in the surface. Figure 7(a) shows a plot of the average roughness of the dosed and annealed GaAs. This plot shows that the average roughness, which is both an indicator of the increased step density and the amplitude of the height variation within the irradiated regions, is constant for $D<10^{14}$ ions $\mathrm{cm}^{-2}$ and increases for $D>$ $10^{14}$ ions $\mathrm{cm}^{-2}$. This is expected, as the amount of material expected to sputter from the surface increases with increasing ion dose [17].

The 2D islands are visible within the treated region shown in figure 6 that are aligned parallel to the raster direction of the FIB during patterning, forming stripes. This is typical for regions irradiated with ion doses $D \approx 10^{14}$ ions $\mathrm{cm}^{-2}$. It is interesting to note that in these regions the stripe width is two to six times wider than the pixel spacing of $30 \mathrm{~nm}$, which suggests that there is significant coarsening of the pattern upon annealing. Furthermore, the stripe width decreases with increasing dose. Despite the formation of stripes, the overall vicinality of the surface is not changed, and there is only a weak alignment of the quantum dots that are grown on samples that contain these surface stripes. Instead, the subsequent deposition of quantum dots upon these stripes results in a random distribution across the patterned region.

In addition to the increase in step density, small holes develop randomly across the GaAs surface. The holes do
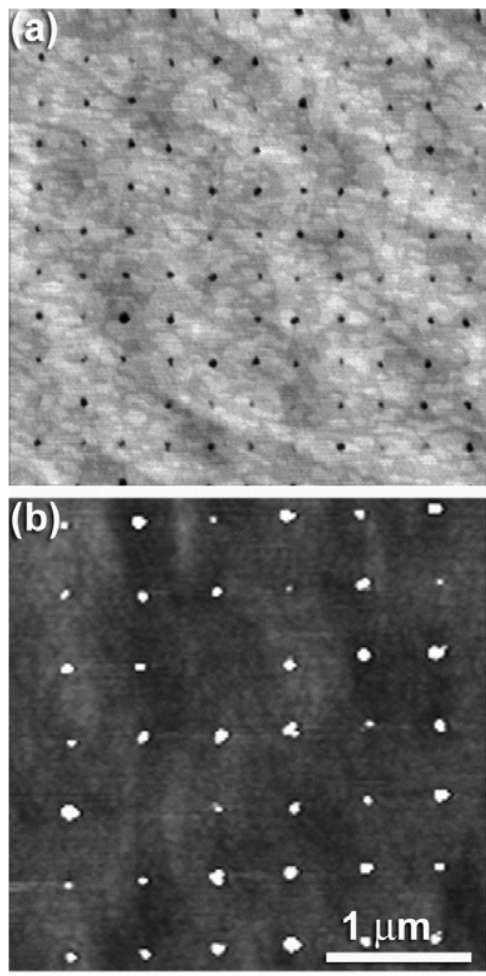

Figure 8. AFM images of (a) a GaAs buffer layer that has been patterned with an array of holes spaced $250 \mathrm{~nm}$ apart and dosed with a dwell time of $800 \mu$ s and (b) a 2 ML InAs film deposited on the pattern spaced $500 \mathrm{~nm}$ apart and dosed with a dwell time of $800 \mu \mathrm{s}$. The height scale is $5 \mathrm{~nm}$ in (a) and $35 \mathrm{~nm}$ in (b).

not vary in size as a function of ion dose, having an average diameter of $11 \pm 6$ and $0.65 \pm 0.06 \mathrm{~nm}$ depth; however, there is a dependence of their density on ion dose. Figure 7(b) shows a plot of the density of holes across the surface. As with the dot density, the density of holes rapidly increases to a value of $N=10^{10} \mathrm{~cm}^{-2}$ at $D>10^{14}$ ions $\mathrm{cm}^{-2}$, and saturates. In fact, the value of the hole density as a function of ion dose is virtually identical to that of the quantum dot density for the 1.7 ML InAs film (see figure 2). This indicates that enhanced quantum dot formation is due to the presence of the ion induced holes, as opposed to a higher density of step edges. These results are also in general agreement with recent results from Hull and co-workers [24, 25], that show the preferential nucleation of Ge quantum dots on FIB-induced surface holes. As in those results, the holes caused by the ion beam can be used to produce regular arrays of quantum dots. Figure 8 shows AFM images of (a) a GaAs buffer layer that has been patterned with an array of holes spaced $250 \mathrm{~nm}$ apart and dosed with a dwell time of $800 \mu \mathrm{s}$, followed by an anneal to $T=530^{\circ} \mathrm{C}$. For this set of patterning conditions, the holes are $35 \pm 13 \mathrm{~nm}$ in diameter and $1.0 \pm 0.1 \mathrm{~nm}$ deep. Figure 8(b) shows an AFM micrograph of a 2.0 ML InAs film deposited at $T=530{ }^{\circ} \mathrm{C}$ upon a similar pattern, having a spacing of $500 \mathrm{~nm}$ and a dwell time of $800 \mu \mathrm{s}$. Dots having an average diameter of $58 \pm 19 \mathrm{~nm}$ and height of $12 \pm 5 \mathrm{~nm}$ nucleate upon the holes, resulting in a fill factor of $98 \%$ over a $9 \mu \mathrm{m}^{2}$ region, demonstrating the excellent fidelity of this technique for the placement of quantum dots in specific locations. 


\section{Conclusions}

We have shown that low doses of uniform irradiation of GaAs substrates by a focused beam of $30 \mathrm{keV} 7.5 \mathrm{pA} \mathrm{Ga}^{+}$alter the subsequent nucleation and growth of of InAs quantum dots. Quantum dots may be induced in patterned regions despite the fact that the deposited thickness is less than the critical thickness for their formation under typical growth conditions. The threshold dose for modifying the quantum dot density is $10^{13}$ ions $\mathrm{cm}^{-2}$, and the dot density increases with increasing ion dose, and reaches saturation for $D>10^{14}$ ions $\mathrm{cm}^{-2}$. This increase is primarily a result of diffusion of adatoms from outside the patterned region. We also show that the mechanism for enhanced quantum dot formation is due to the formation of monolayer deep holes created in the substrate by the ion beam. The advantages of this technique for dots nucleated on uniformly dosed regions are excellent area selectivity and the ability to control the dot density, a feature potentially useful for optical intensity control in light emitting devices or in tandem solar cells with quantum dots in the active region. Further measurements to evaluate the ion beam-induced defects on the optical properties are planned, but comparisons of annealed and as-grown features suggest that much of the ion beam damage is removed upon annealing.

\section{References}

[1] Eberl K, Lipinski M O, Manz Y M, Winter W, Jin-Phillipp N Y and Schmidt O G 2001 Physica E 9164

[2] Livshits D A, Kovsh A R, Maleev N A, Zhukov A E, Ustinov V M, Ledentsov N N, Alferov Z I, Bimberg D, Lin G and Chi J 2003 Proc. SPIE 4999524

[3] Solomon G S, Pelton M and Yamamoto Y 2002 Proc. SPIE 465649

[4] Kiravittaya S, Songmuang R, Rastelli A, Heidemeyer H and Schmidt O G 2006 Nanoscale Res. Lett. 11

[5] Lee H, Johnson J A, Speck J S and Petroff P M 2000 J. Vac. Sci. Technol. B 182193

[6] Watanabe S, Pelucchi E, Leifer K, Malko A, Dwir B and Kapon E 2005 Appl. Phys. Lett. 86243105
[7] Tranvouez E, Gendry M, Regreny P and Bremond G 2004 Superlatt. Microstruct. 36325

[8] Song H Z, Nakata Y, Okada Y, Miyazawa T, Ohshima T, Takatsu M, Kawabe M and Yokoyama N 2004 Physica E 21625

[9] Kapsa J, Robach Y, Hollinger G, Gendry M, Gierak J and Mailly D 2004 Appl. Surf. Sci. 22631

[10] Hull R, Gray J L, Kammler M, Vandervelde T, Kobayashi T, Kumar P, Pernell T, Bean J C, Floro J A and Ross F M 2003 Mater. Sci. Eng. B 1011

[11] Karmous A, Cuenat A, Ronda A, Berbezier I, Atha S and Hull R 2004 Appl. Phys. Lett. 856401

[12] Kubis A J, Vandervelde T E, Bean J C, Dunn D N and Hull R 2004 Nanoparticles and Nanowire Building Blocks-Synthesis, Processing, Characterization and Theory p 411

[13] Sun Y T, Messmer E R, Lourdudoss S, Ahopelto J, Rennon S, Reithmaier J P and Forchel A 2001 Appl. Phys. Lett. 791885

[14] Morishita Y, Ishiguro M, Miura S and Enmel Y 2002 J. Cryst. Growth 237-239 1291

[15] Kim Y K, Danner A J, Raftery J J and Choquette K D 2005 IEEE J. Sel. Top. Quantum Electron. 111292

[16] Kitslaar P, Strassner M, Sagnes I, Bourhis E, Lafosse X, Ulysse C, David C, Jede R, Bruchhaus L and Gierak J 2006 Microelectron. Eng. 83811

[17] Menzel R, Bachmann A T and Wesch W 1999 Nucl. Instrum. Methods B 148450

[18] Lugstein A, Basnar B and Bertagnolli E 2002 J. Vac. Sci. Technol. B 202238

[19] Basnar B, Lugstein A, Wanzenboeck H, Langfischer H, Bertagnolli E and Gornik E 2003 J. Vac. Sci. Technol. B 21927

[20] Vandervelde T E, Atha S, Hull R, Pernell T L and Bean J C 2006 J. Vac. Sci. Technol. A 24375

[21] Kammler M, Hull R, Reuter M C and Ross F M 2003 Appl. Phys. 821093

[22] Lee J H, Wang Z M, Liang B L, Sablon K A, Strom N W and Salamo G J 2006 Semicond. Sci. Technol. 211457

[23] Wang Zh M, Lee J H, Liang B L, Black W T, Kunets V P, Mazur Yu I and Salamo G J 2006 Appl. Phys. Lett. 88233102

[24] Portavoce A, Kammler M, Hull R, Reuter M C and Ross F M 2006 Nanotechnology 174451

[25] Gray J L, Hull R and Floro J A 2006 J. Appl. Phys. 10084312 